\title{
Experimental Validation of a Robust Continuous Nonlinear Model Predictive Control Based Grid-Interlinked Photovoltaic Inverter
}

\author{
Rachid Errouissi, Member, IEEE, S. M. Muyeen, Senior Member, IEEE, Ahmed Al-Durra, Senior Member, IEEE, \\ and Siyu Leng Member, IEEE
}

\begin{abstract}
The paper presents a robust continuous nonlinear model predictive control (CNMPC) for a grid-connected photovoltaic (PV) inverter system. The objective of the proposed approach is to control the power exchange between the grid and a photovoltaic system, while achieving unity power factor operation. As the continuous nonlinear MPC cannot completely remove the steady-state error in the presence of disturbances, the nonlinear disturbance observer-based control is adopted to estimate the offset caused by parametric uncertainties and external perturbation. The stability of the closed-loop system under both nonlinear predictive control and disturbance observer is ensured by convergence of the output-tracking error to the origin. The proposed control strategy is verified using a complete laboratoryscale PV test-bed system consisting of a photovoltaic emulator, a boost converter, and a grid-tied inverter. High performance with respect to DC-link voltage tracking, grid current control, disturbance rejection, and unity power factor operation has been demonstrated.
\end{abstract}

\section{INTRODUCTION}

$\mathbf{M}$ ODEL Predictive Control (MPC) is now regarded as one of the most promising approaches in control engineering due to the drastic improvements in the processors capabilities. The idea behind the MPC is to find an optimal control minimizing a cost function whose form depends on the performance specifications. Thus the MPC can be formulated as an optimization problem [1].

A look at the literature reveals that MPC has been extensively applied in power converters [2]-[8] and the DiscreteTime Linear Model (DTLM) is widely used in MPC synthesis because it gives a fast analytical solution of the optimization problem. For power converters, the most popular MPC scheme, known as finite control set model predictive control (FCS-MPC), evaluates a cost function for all possible voltage vectors, and selects the one which gives the lowest cost function value [5].

In recent years, this technique has been successfully applied in power converters for renewable energy systems, where the

Manuscript received April 13, 2015; revised August 18, 2015; October 18, 2015; accepted November 20, 2015.

Copyright (C)2015 IEEE. Personal use of this material is permitted. However, permission to use this material for any other purposes must be obtained from the IEEE by sending a request to pubs-permissions@ieee.org.

This work was supported by "The Petroleum Institute Research Center (PIRC)" Research Grant. R. Errouissi, S. M. Muyeen, A. Al-Durra, and S. Leng are with the Petroleum Institute, P.O. Box 2533, Abu Dhabi, United Arab Emirates (e-mails: rerrouissi@pi.ac.ae, smmuyeen@pi.ac.ae, aaldurra@pi.ac.ae, and sleng@pi.ac.ae). main control objective is to regulate the DC-link voltage, and the power injected into the grid [9]-[14]. In [15], a predictive control is implemented to realize maximum power point tracker algorithm, where the main work is concerned with a stand-alone photovoltaic system. Although satisfactory results can be obtained with such a strategy, a precise knowledge of the model is required to obtain accurate prediction of the system behavior. Another shortcoming is that a significant computational effort is still required to determine the optimal switching states, in particular, for power converters with higher levels. However, the main drawback lies in the use of high and variable switching frequency, which raises concern about switching losses in semiconductor devices and feasibility for medium and high power applications.

In the aforementioned works, Discrete-Time model (DTM) is used to predict the system behavior, whereas the three-phase grid-connected inverter can be approximated by a continuoustime nonlinear model, and the use of DTM may not be the right choice for the controller design. However, it is still a challenge to apply MPC for nonlinear systems having fast dynamics, such as adjustable electrical drives and power converters used in renewable energy and power system applications due to the heavy on-line computational burden. Over the last few years, several nonlinear predictive control laws have been proposed to reduce the computational effort. The majority of these works are based on continuous time affine nonlinear model [16]-[22], where the prediction model is obtained by the use of Taylor series expansion. A methodology for constructing closedform nonlinear MPC is proposed in [16]-[18], where the one step ahead approach is used to predict the future system output by using Taylor series expansion up to the relative degree. The multi-step ahead prediction approach is introduced in [19], where it is used to approximate the nonlinear receding horizon control problem. As pointed out in [18], predictive control based on Taylor series expansion cannot guarantee zero steady-state error in the presence of model uncertainty and external perturbation. In this context, a disturbance observer has been proposed in [20], combining the CNMPC in order to compensate for the offsets. That work is extended to mutliinput multi-output (MIMO) system in [21]. In [22], it was shown that the closed-loop stability is guaranteed for nonlinear systems having the relative degree less than or equal to four.

Nowadays, the closed-form CNMPC becomes more mature and there exists a steadily increasing interest in real time implementation of this technique to nonlinear systems with fast 
dynamics such as electrical drives and power converters [23]. In [24], a composite controller consisting of CNMPC and a disturbance observer is implemented in real-time to a permanent magnet synchronous motor, and improved performance is reported. In CNMPC, the resulting control is closely related to feedback linearization [25]. Hence, in the presence of unknown disturbances and parameter uncertainties, the system output tracks its reference signal with an error which does not die out completely. Clearly, this is the effect of the disturbances that need to be decoupled from the system output, which is still a challenge problem for unmatched disturbances.

To overcome this problem, a mathematical model that does not exhibit unmatched disturbances is utilized in [26] to design a feedback-linearization for three-phase grid-connected photovoltaic inverter system, where only simulation tests are considered. Relying on the assumption that the matching conditions are satisfied, a feedback linearization is practically implemented to three-phase grid-connected photovoltaic system in [27], where the boost converter is not used, and the maximum power point tracking (MPPT) is achieved by adjusting the DC-link voltage, therefore, the real PV dynamics is not considered. The shortcoming of the work presented in [26], [27] is that the closed-loop system exhibits zero dynamics, which requires stability analysis. To overcome such a drawback, another model is adopted in [28], where the grid-connected PV system is fully linearized, which means that there is no zero dynamics. In this case, the disturbance appearing in the model is not necessarily matched and can be considered as unmatched, in particular, in the absence of the information about the PV current. In the aforementioned works, integral action is employed to cancel the effect of model uncertainty, and the PV current is assumed to be available for measurement, which is not always true. Although, the measurement of PV current can be available, a low pass filter is required to minimize the current ripples caused by the boost converter. In [29], the disturbance observer is experimentally implemented to estimate the power disturbances in the DC sub-grid such as the battery and the PV unit, where only few realistic scenarios were performed. Such a disturbance observer is used in a recent work [30], to recover the nominal performance of electric motor drives under model uncertainties and external disturbances.

In this paper, a continuous nonlinear model predictive control (CNMPC) is combined with a disturbance observer to practically control a grid-interlinked photovoltaic system in real time using a laboratory test-bed consisting of a PV unit, a boost converter, a DC-link capacitor, an inverter, and a line filter. Such a control scheme does not require the current information of the PV unit, which appears in the model as unmatched disturbance. More specifically, it turns out that the disturbance observer can be used to cancel the effect of all parameter variation and external disturbance, whether matched or unmatched. More interestingly, unlike FCS-MPC scheme, the proposed approach can operate at a low and constant switching frequency without compromising the power quality. The control objective is to regulate the DC-link voltage while maintaining the quadrature-axis grid current equal to zero to ensure unity power factor operation.
The paper is organized as follows. In Sect. II, the model of the three-phase grid-connected photovoltaic system is presented. CNMPC is developed in Sect. III for the system under investigation. Sections IV, V and VI give details of the Laboratory setup, controller implementation, and experimental results, respectively. The conclusion is drawn in Sect. VII.

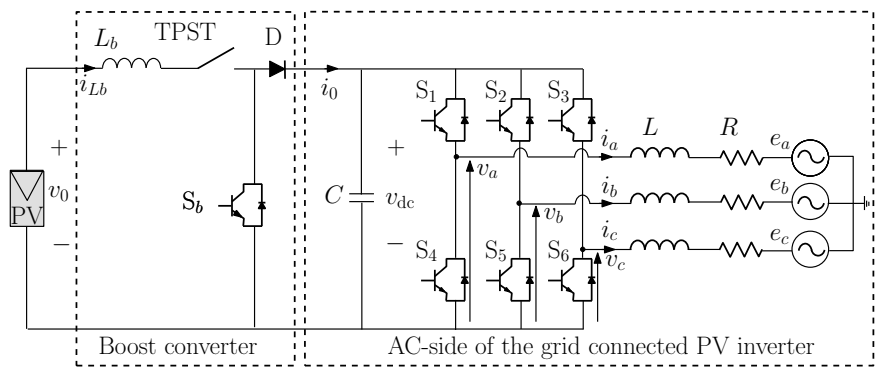

Fig. 1. Schematic diagram of a grid-interlinked photovoltaic inverter system.

\section{Mathematical MODEL}

The schematic diagram of the grid-interlinked PV system investigated in this work is shown in Fig. 1. The PV output voltage $v_{0}$ is regulated by adjusting the duty cycle $d$ of the boost converter, so that the PV system operates at a specified power point, e.g., maximum power point. The DC-link voltage $v_{d c}$ is viewed as an input to the boost converter and is regulated through the control of the grid-side inverter. The control of the AC-side PV inverter represents the main focus of this paper. The general model of the grid-side converter is detailed in [28]. Following the steps shown in [31], one can use the model described in [28] to write the perturbed model in the nonlinear affine form as follows

$$
\left\{\begin{array}{c}
\dot{x}=f(x)+g_{1} u(t)+g_{2} b(t) \\
y_{i}=h_{i}(x), i=1,2
\end{array}\right.
$$

where the state vector $x$ consists of $d$-axis current $i_{d}, q$-axis current $i_{q}$, and DC-link voltage $v_{d c}$, and the input vector $u$ is made of $d$-axis and $q$-axis components of the inverter voltage as follows

$$
x=\left[\begin{array}{lll}
i_{d} & i_{q} & v_{d c}
\end{array}\right]^{T} \quad, \quad u=\left[\begin{array}{ll}
v_{d} & v_{q}
\end{array}\right]^{T}
$$

The vector field $f(x)$ is given by

$$
f(x)=\left[\begin{array}{c}
-\frac{R}{L} i_{d}+\omega i_{q}-\frac{e_{d}}{L} \\
-\frac{R}{L} i_{q}-\omega i_{d}-\frac{e_{q}}{L} \\
-\frac{3}{2 C v_{d c}}\left(e_{d} i_{d}+e_{q} i_{q}\right)
\end{array}\right]
$$

and $g_{1}$ and $g_{2}$ are defined as

$$
g_{1}=\left[\begin{array}{ll}
g_{d} & g_{q}
\end{array}\right]=\left[\begin{array}{cc}
\frac{1}{L} & 0 \\
0 & \frac{1}{L} \\
0 & 0
\end{array}\right], g_{2}=\left[\begin{array}{ccc}
\frac{1}{L} & 0 & 0 \\
0 & \frac{1}{L} & 0 \\
0 & 0 & \frac{1}{C}
\end{array}\right]
$$

Here, $R, L$ and $C$, are respectively the filter resistance, the filter inductance and the DC-link capacitor. The variable $\omega$ denotes the angular frequency of the grid voltage. In general, the grid code requires a constant frequency, and only a small frequency variation is allowable to ensure reliable, efficient and secure operation.

It should be noted that the $d q$ model is obtained from $a b c$ 
model by applying $d q$ transformation using a phase-locked loop (PLL) approach. Such an algorithm is used to estimate the angular frequency and the reference angle for the rotating reference frame based on the voltage measurements. In this work, the design of the three-phase PLL relies on the assumption that there are no zero sequence components and the grid is balanced. The PLL structure proposed in [32], is used as a basis to estimate the reference angle, so as to guarantee $e_{q}=0$ in the steady-state regime, meaning that $d$-axis is chosen to be aligned with the fundamental of the grid voltage. The structure of the PLL based on synchronous reference frame is depicted in Fig. 2. The idea is to use a proportional-integrator (PI) controller to achieve zero $q$-axis grid voltage. A small signal analysis using the linearized model of the three-phase PLL is employed to tune the parameters of the PI controller according to the performance specifications [33].

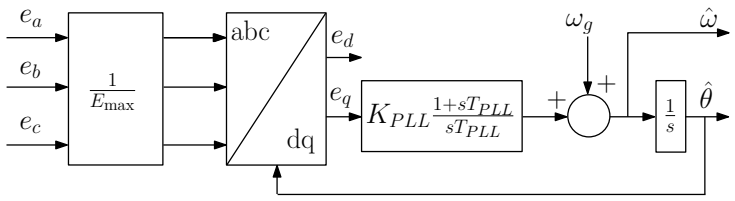

Fig. 2. Structure of the PLL used to synchronize the PV system with the grid, with $\omega_{g}$ and $E_{\max }$ are, respectively, the nominal angular frequency and the peak value of the grid voltage. The PI controller parameters are as follows: $K_{P L L}=92$ and $T_{P L L}=0.0217 \mathrm{~s}$ [33].

Using the above structure of the PLL, the reactive power control can be implemented through control of $q$-axis current. Hence, the variables to be controlled are $q$-axis current and DC-link voltage, which implies

$$
y_{1}=i_{q} \quad, \quad y_{2}=v_{d c}
$$

The disturbance vector $b(t)=\left[\begin{array}{lll}b_{d} & b_{q} & b_{v}\end{array}\right]^{T}$ represent perturbation resulting from parameter variations $\Delta R, \Delta L$ and $\Delta C$, and uncertainties in grid voltage measurement $e_{d}$ and $e_{q}$, as well as the unknown external disturbance $i_{0}$ generated by photovoltaic system. It follows that the disturbances can be expressed as

$$
\left\{\begin{array}{l}
b_{d}=\Delta R i_{d}-\omega \Delta L i_{q}+\Delta e_{d}+\Delta L \frac{d i_{d}}{d t}+\delta_{d} \\
b_{q}=\Delta R i_{q}+\omega \Delta L i_{q}+\Delta e_{q}+\Delta L \frac{d i_{q}}{d t}+\delta_{q} \\
b_{v}=\frac{3}{2 v_{d c}}\left(\Delta e_{d} i_{d}+\Delta e_{q} i_{q}\right)+i_{0}+\Delta C \frac{d v_{d c}}{d t}+\delta_{v}
\end{array}\right.
$$

where the variables $\delta_{d}, \delta_{q}$, and $\delta_{v}$ can be considered as unknown external disturbances acting on the system, such as the PWM offset and the angular frequency variation. Furthermore, as the control input is represented by $v_{d}$ and $v_{q}$, the nonlinear system is with both matched and unmatched disturbances. Here, unmatched disturbance $b_{v}$ depends on the PV current $i_{0}$, which is considered as unknown variable. Additionally, the PV current contains high frequency component due to the boost converter.

Assumption: The states are assumed to have constant steadystate. Following (6), and considering only the dc component of the current $i_{0}$, it can be assumed that the disturbance is bounded, and satisfies

$$
\lim _{t \rightarrow \infty} \dot{b}=0
$$

Knowing the nominal value of the current $i_{d}$, the boundaries of the disturbance, at steady-state, can be numerically approximated by substituting all possible combinations of the extreme values of $\Delta R, \Delta L, \Delta C, \Delta e_{d}, \Delta e_{q}$ and $i_{0}$ into the vector $b$ defined by (6). However, unlike some existing robust controllers, such bounds are not required to enhance the disturbance rejection capability, which reduces the design complexity.

\section{CONTINUOUS NONLINEAR PREDICTIVE CONTROL}

\section{A. Formulation of the control law}

As in [22], continuous CNMPC seeks a control signal which minimizes a quadratic cost function consisting of the difference between the system output $y$ and the trajectory to be tracked $y_{r}$. For the system under investigation, the cost function can be written as follows

$$
\Im=\frac{1}{2} \int_{0}^{T_{1}} q_{1}^{2} e_{1}(t+\tau)^{2} d \tau+\frac{1}{2} \int_{0}^{T_{2}} q_{2}^{2} e_{2}(t+\tau)^{2} d \tau
$$

where $q_{1}$ and $q_{2}$ are the weighting parameters and $T_{i}$ is the predictive time for the output $y_{i}$. The tracking errors $e_{1}$ and $e_{2}$ are expressed as follows

$$
\left\{\begin{array}{l}
e_{1}(t+\tau)=i_{\text {qref }}(t+\tau)-i_{q}(t+\tau) \\
e_{2}(t+\tau)=v_{\text {dcref }}(t+\tau)-v_{d c}(t+\tau)
\end{array}\right.
$$

It is worth noting that by using two different predictive times, it is possible to combine current and voltage into a single cost function although their dynamics are different. On the other hand, it can be shown that the resultant optimal control does not depend on the values of $q_{1}$ and $q_{2}$ [23]. Therefore, one can choose $q_{1}=1$ and $q_{2}=1$ although both outputs are with different magnitudes. The optimal control can be obtained from the necessary condition of optimality

$$
\frac{d \Im}{d u}=0
$$

To solve the nonlinear optimization problem (8), each predicted term $e_{i}(t+\tau)$, with $i=[1,2]$, is expanded into a $\left(\rho_{i}+r_{i}\right)^{t h}$ order Taylor series expansion using the Lie derivative $h_{i}(x)$ along a field of vectors $f(x)$. Here, $\rho_{i}$ represents the relative degree for each of the outputs, and the standard Lie derivative notation is used [34]. The control order is denoted by $r$, which is chosen as zero in this work in order to simplify the notation. In the continuous nonlinear MPC design method, it is necessary to calculate the relative degree for each output $y_{i}$. The relative degrees for both $i_{q}$ and $v_{d c}$ are respectively $\rho_{1}=1$ and $\rho_{2}=2$. Since, the total relative degree of the system is given by $\rho_{1}+\rho_{2}=3$, and is equal to the system's order, there is no zero dynamics. In addition, the states are assumed available for measurement, which implies that the nonlinear system (1) is input-output feedback linearizable if the relative degree is well-defined. This can be verified by showing that the following matrix

$$
\begin{aligned}
G(x) & =\left[\begin{array}{cc}
L_{g_{d}} h_{1}(x) & L_{g_{q}} h_{1}(x) \\
L_{g_{d}} L_{f} h_{2}(x) & L_{g_{q}} L_{f} h_{2}(x)
\end{array}\right] \\
& =\left[\begin{array}{cc}
0 & \frac{1}{L} \\
-\frac{3 e_{d}}{2 L C v_{d c}} & -\frac{3 e_{q}}{2 L C v_{d c}}
\end{array}\right]
\end{aligned}
$$

is nonsingular, at least locally. Indeed, one can remark that $G(x)$ is invertible if $v_{d c}$ and $e_{d}$ are different from zero. 
In practice, $v_{d c}$ and $e_{d}$ cannot be equal to zero for the three-phase grid-connected photovoltaic system. Hence, the nonlinear system (1) is input-output feedback linearizable. Now, according to the definition of the relative degree, one can write

$$
\left\{\begin{array}{l}
\dot{y}_{1}(t)=L_{f} h_{1}(x)+L_{g_{1}} h_{1}(x) u+L_{g_{2}} h_{1}(x) b \\
\dot{y}_{2}(t)=L_{f} h_{2}(x)+L_{g_{2}} h_{2}(x) b
\end{array}\right.
$$

where

$$
L_{g_{2}} h_{2}(x) b=\frac{b_{v}}{C}
$$

Considering (13), $\ddot{y}_{2}$ can be expressed as

$$
\ddot{y}_{2}(t)=L_{f}^{2} h_{2}(x)+L_{g_{1}} L_{f} h_{2}(x) u+L_{g_{2}} L_{f} h_{2}(x) b+\frac{\dot{b}_{v}}{C}
$$

It can be shown (see the Appendix A) that the continuous nonlinear predictive control law is expressed as

$$
u=G^{-1}(x)\left(H(x)-M(x) b-\left[\begin{array}{c}
0 \\
\frac{1}{C}
\end{array}\right] \dot{b}_{v}\right)
$$

where

$$
H(x)=\left[\begin{array}{c}
\sum_{j=0}^{1} K_{j}^{1}\left(i_{\text {qref }}^{(j)}-L_{f}^{j} h_{1}(x)\right) \\
\sum_{j=0}^{2} K_{j}^{2}\left(v_{\text {dcref }}^{(j)}-L_{f}^{j} h_{2}(x)\right)
\end{array}\right]
$$

and

$$
\begin{aligned}
& M(x)= {\left[\begin{array}{c}
M_{1}(x) \\
M_{2}(x)
\end{array}\right]=\left[\begin{array}{c}
L_{g_{2}} h_{1}(x) \\
K_{1}^{2} L_{g_{2}} h_{2}(x)+L_{g_{2}} L_{f} h_{2}(x)
\end{array}\right] } \\
&=\left[\begin{array}{ccc}
0 & \frac{1}{L} & 0 \\
\frac{-3 e_{d}}{2 C L v_{d c}} & 0 & \left(\frac{K_{1}^{2}}{C}+\frac{-3 e_{d} i_{d}}{2 C^{2} v_{d c}^{2}}\right)
\end{array}\right]
\end{aligned}
$$

The controller gains $K_{0}^{1}, K_{1}^{1}, K_{0}^{2}, K_{1}^{2}$ and $K_{2}^{2}$ are given by

$$
\left\{\begin{array}{l}
K_{0}^{1}=\frac{3}{2 T_{1}}, K_{1}^{1}=1 \\
K_{0}^{2}=\frac{10}{3 T_{2}^{2}}, K_{1}^{2}=\frac{5}{2 T_{2}}, K_{2}^{2}=1
\end{array}\right.
$$

As the predictive times $T_{1}$ and $T_{2}$ are positives, It can easily be shown that the polynomials

$$
\left\{\begin{array}{l}
K_{1}^{1} s+K_{0}^{1}=0 \\
K_{2}^{2} s^{2}+K_{1}^{2} s+K_{0}^{2}=0
\end{array}\right.
$$

are Hurwitz.

In the case of a step response, it can be shown that the current loop is a typical first-order system; with a time constant equal to $0.66 \times T_{1}$, while the closed-loop system for the DClink voltage control is a second-order system, whose natural angular frequency $\omega_{n}$, damping ratio $\xi$, and settling time $t_{s}$ are given by

$$
\omega_{n}=1.82 T_{2}^{-1} \mathrm{rad} / \mathrm{s}, \quad \xi=0.68, t_{s}=3.28 T_{2} \mathrm{~s}
$$

Therefore, the predictive times $T_{1}$ and $T_{2}$ can be considered as tunable parameters, and can be chosen according to the closedloop performance specifications. The step response overshoot is constant and is equal to $5.23 \%$. To limit the current during transients, the step response should be avoided. For this purpose, the DC-link voltage reference should be chosen in adequate way, so that the current does not exceed its maximal value during transients.

Clearly the resulting control is closely related to feedback linearization, but the design method/philosophy is different. It should be noted that the controller is difficult to practical implement as the disturbance and its time derivative are not available for measurement. However, as the time derivative of the disturbance vanishes as time goes to infinity, one can simplify the proposed controller as follows

$$
u=G^{-1}(x)\left(\left[\begin{array}{c}
\sum_{j=0}^{1} K_{j}^{1}\left(i_{q r e f}^{(j)}-L_{f}^{j} h_{1}(x)\right) \\
\sum_{j=0}^{2} K_{j}^{2}\left(v_{\text {dcref }}^{(j)}-L_{f}^{j} h_{2}(x)\right)
\end{array}\right]-M(x) b\right)
$$

Substituting the state feedback control law (21) into (12)-(14) gives the closed-loop system error equation as follows

$$
\left\{\begin{array}{l}
\dot{e}_{1}+K_{0}^{1} e_{1}=0 \\
\ddot{e}_{2}+K_{1}^{2} \dot{e}_{2}+K_{0}^{2} e_{2}=-\frac{\dot{b}_{v}}{C}
\end{array}\right.
$$

The stability of the closed-loop error can be established by invoking the polynomials defined by (19). In fact, since the time derivative of the disturbance $b$ is bounded, then the closed-loop system error (22) is input-to-state stable (ISS) with respect to the disturbance $\dot{b}$ [34]. In addition, with the assumption that the time derivative of the disturbance vanishes as time goes to infinity, one can conclude that the output tracks its reference with an error which satisfies

$$
\lim _{t \rightarrow \infty} e_{1}(t)=0, \quad \lim _{t \rightarrow \infty} e_{2}(t)=0
$$

The derivation of the proposed controller is based on the assumption that all variables, including the angular frequency, are available for measurement. This means that the closedloop system error equation (22) is still valid even if the angular frequency varies. Therefore, any change in the angular frequency will not have an impact on the steady-state performances. Moreover, it is worth noting that, practically, the angular frequency is maintained constant by the electrical power grid to comply with the grid code.

Following (21) the information about the disturbances is required to practically implement the state feedback control law. To address this problem, the disturbance observer, proposed in [20] and combined with sliding mode control in [35], will be used as a basis for estimating the unknown perturbation terms $b$ acting on the system.

\section{B. Design of the disturbance observer}

As all states are assumed to be available, the disturbance $b(t)$ is supposed to be observable. Thus, the disturbance in (21) can be replaced by its estimate $\hat{b}$. This can be accomplished by designing an appropriate estimator. For the three-phase grid-connected photovoltaic system, an initial disturbance observer is given by

$$
\dot{\hat{b}}=-l(x) g_{2} \hat{b}+l(x)\left(\dot{x}-f(x)-g_{1} u\right)
$$

where $l(x)$ represents the observer gain, and it is given by

$$
l(x)=\frac{\partial p(x)}{\partial x}
$$


with $p(x)$ is a nonlinear function. It follows that the disturbance estimation error is governed by

$$
\dot{\hat{b}}-\dot{b}=-l(x) g_{2}(\hat{b}-b)-\dot{b}
$$

Now, if $p(x)$ is chosen as

$$
p(x)=\left[\begin{array}{lll}
\mu_{d} i_{d} & \mu_{q} i_{q} & \mu_{v} v_{d c}
\end{array}\right]^{T}
$$

where $\mu_{d}, \mu_{q}$ and $\mu_{v}$ are constant observer gains. Then, substituting (25) into (26) with (27) yields

$$
\dot{e}_{p}=-\operatorname{diag}\left\{\mu_{d}, \mu_{q}, \mu_{v}\right\} g_{2} e_{p}-\dot{b}=-\mu g_{2} e_{p}-\dot{b}
$$

where $e_{p}$ represents the disturbance estimation error, and it is given by

$$
e_{p}=\hat{b}-b
$$

Therefore, as $\dot{b}$ is bounded and the elements of $g_{2}$ are positives, then it can easily be proved that the observer error system (28) is ISS, with respect to the disturbance $\dot{b}$, if the observer gains $\mu_{d}, \mu_{q}$ and $\mu_{v}$ are chosen positives. Furthermore, provided that the time derivative of the disturbance is asymptotically vanishing, the asymptotic stability of the disturbance observer can be guaranteed. The convergence rate of the disturbance observer depends on the observer gain $\mu$. Indeed, the time constant for the matched disturbances $b_{d, q}$ is equal to $L / \mu_{d, q}$, whereas the time constant for unmatched disturbance $b_{v}$ is equal to $C / \mu_{v}$. Thereby, a small observer gain results in a slow convergence rate. Generally, the dynamic response of the observer should be much faster than that of the controller, but a very large observer gain may accentuate the measurement noises. From (28), it can be observed that the disturbance observer is equivalent to a first-order lowpass filter, where the cut-off frequency is determined by the time constant of the observer. Therefore, a smaller observer gain will guarantee a smooth estimate. For real time implementation, the practical issue is that the time derivative of the state is not available for measurement. To overcome this drawback, one can replace the initial disturbance observer by its equivalent one as follows

$$
\left\{\begin{array}{l}
\dot{\dot{ }} z=-l(x) g_{2}(p(x)+\hat{z})-l(x)\left(f(x)+g_{1} u\right) \\
\hat{b}=\hat{z}+p(x)
\end{array}\right.
$$

where $z$ is an auxiliary variable to be estimated. In the absence of disturbances, the initial state of the disturbance observer (30) can be chosen as $z(0)=-p(x(0))$. Under these conditions, the nominal performances can be preserved in the absence of disturbances.

\section{Stability analysis for the composite controller}

By replacing the disturbance $\mathrm{b}$ by its estimate $\hat{b}$, and substituting the control law (21) into (12)-(14), leads to the augmented closed-loop error system

$$
\left\{\begin{array}{l}
\dot{e}_{1}=-K_{0}^{1} e_{1}-M_{1}(x) e_{p} \\
\ddot{e}_{2}=-K_{1}^{2} \dot{e}_{2}-K_{0}^{2} e_{2}-M_{2}(x) e_{p}-\frac{\dot{b}_{v}}{C} \\
\dot{e}_{p}=-\mu g_{2} e_{p}-\dot{b}
\end{array}\right.
$$

where

$$
M_{1}(x)=M_{1}=\left[\begin{array}{lll}
0 & \frac{1}{L} & 0
\end{array}\right]
$$

and

$$
M_{2}(x)=\left[\begin{array}{lll}
\frac{-3 e_{d}}{2 C L v_{d c}} & 0 & \frac{1}{C}\left(K_{1}^{2}+\frac{3 e_{d} i_{d}}{2 C v_{d c}^{2}}\right)
\end{array}\right]
$$

It follows from (1)-(3) that

$$
\frac{3 e_{d} i_{d}}{2 C v_{d c}^{2}}=\frac{1}{v_{d c}}\left(\dot{v}_{d c}-\frac{b_{v}}{C}\right)
$$

Note that

$$
v_{d c}=v_{d c r e f}-e_{2}, \quad \dot{v}_{d c}=\dot{v}_{d c r e f}-\dot{e}_{2}
$$

Therefore, $M_{2}(x)$ only depends on errors $e_{2}, \dot{e}_{2}$ and references $v_{d c r e f}$ and $\dot{v}_{d c r e f}$.

Let $\eta=\left[\begin{array}{lll}e_{1} & e_{2} & \dot{e}_{2}\end{array}\right]^{T}$, and then the augmented closed-loop error system can be rewritten as

$$
\left\{\begin{array}{l}
\dot{\eta}=F\left(\eta, e_{p}\right)+\left[\begin{array}{lll}
0 & 0 & -\dot{b}_{v}
\end{array}\right]^{T} \\
\dot{e}_{p}=-\mu g_{2} e_{p}-\dot{b}
\end{array}\right.
$$

where

$F\left(\eta, e_{p}\right)=\left[\begin{array}{ccc}-K_{0}^{1} & 0 & 0 \\ 0 & 0 & 1 \\ 0 & -K_{0}^{2} & -K_{1}^{2}\end{array}\right]-\left[\begin{array}{c}M_{1} \\ 0 \\ M_{2}\left(e_{2}, \dot{e}_{2}\right)\end{array}\right]_{(37)} e_{p}$

Following Corollary 10.3.2 in [36], which is dedicated to the local asymptotic stability of cascade-connected systems, one can show that the origin of the system (36), with $\dot{b}=0$, is locally asymptotically stable. Indeed, provided that the polynomials defined by (19) are Hurwitz, the origin $\eta=0$ of $\dot{\eta}=F(\eta, 0)$, with $\dot{b}=0$, is locally asymptotically stable. The origin $e_{p}=0$ of $\dot{e_{p}}=-\mu g_{2} e_{p}$, with $\dot{b}=0$, can be made asymptotically stable by an appropriate choice of the observer gain $\mu$. Thereby, since the time derivative of the disturbance $b$ is bounded, then the closed-loop system error (36) is locally input-to-state stable with respect to $\dot{b}$. With the assumption that $\dot{b}$ vanishes as $t \rightarrow \infty$, one can conclude that the origin of the system (36) converges asymptotically to zero as time goes to infinity.

\section{LABORATORY TEST SETUP}

A laboratory-scale PV test-bed is developed to experimentally verify the validity and the effectiveness of the proposed scheme for a grid-interlinked photovoltaic inverter. The testbed has the real dynamics as it consists of a photovoltaic emulator, a dc-dc boost converter, a grid-interlinked inverter, an $L$ filter, and a step up transformer which connects the system to the Abu Dhabi, UAE power grid. This test-bed setup is depicted in Fig. 3. The XR160-12 power supply module, manufactured by Magna-Power Electronics, and operating at $160 \mathrm{~V}$ output voltage, is used in this experiment as a PV emulator. The grid-interlinked inverter consists of a Semiteach power electronics module (AN-8005) manufactured by Semikron. The system parameters are given in Table II of the Appendix B. The Semiteach power electronics module includes two DC-link capacitors connected in series, threephase IGBT modules with driver circuits, and an extra IGBT with an anti-parallel diode connected in series with a diode. The extra IGBT module is used to build the boost converter with an appropriate design of the inductor. The DS1103 board 
is a powerful and popular tool for rapid control prototyping and drive applications, and used in this study to control the boost converter and grid-tied inverter. It is equipped with Power PC 750GX (Master processor) running at $1 \mathrm{GHz}$, and a Texas Instruments TMS320F240 DSP (slave processor) running at $20 \mathrm{MHz}$. The proposed approach has been implemented on the main processor, whereas the slave unit has been dedicated to the PWM signals generation.

In order to implement the proposed controller, a sampling frequency of $5 \mathrm{kHz}$ is high enough to obtain satisfactorily results. However, in this experiment, the sampling frequency is set equal to $12.5 \mathrm{kHz}$, which is dictated by the control of the boost converter. The switching frequency can be selected as a factor of or equal to the sampling frequency. For a grid-connected PV system, the major concern is the total harmonic distortion (THD) of the current, which must meet the host grid requirements. High switching frequency reduces the current harmonics and allows increasing the bandwidth of the current control loop but often at the expense of the switching losses. Another concern is that, for medium and high power applications, the existing semiconductor devices cannot operate at a high switching frequency. From the aforementioned considerations, the switching frequency of the inverter has been chosen equal to $2.5 \mathrm{kHz}$, which is high enough to have a fast current control and a low THD of the current.

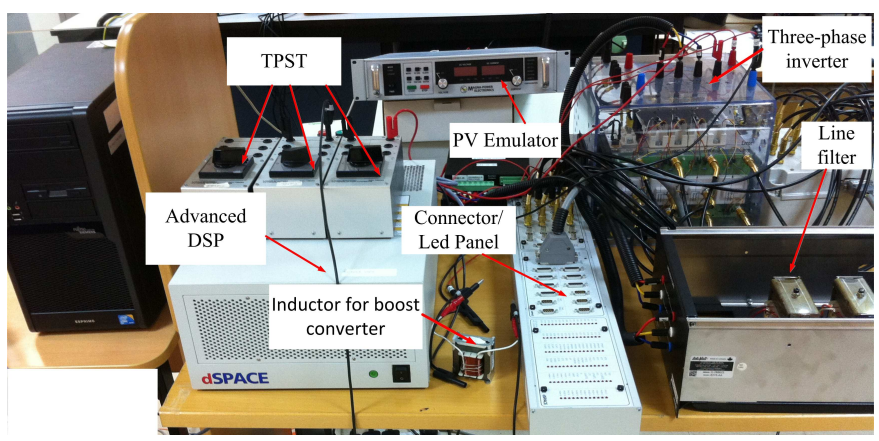

Fig. 3. Experimental setup for PV test-bed.

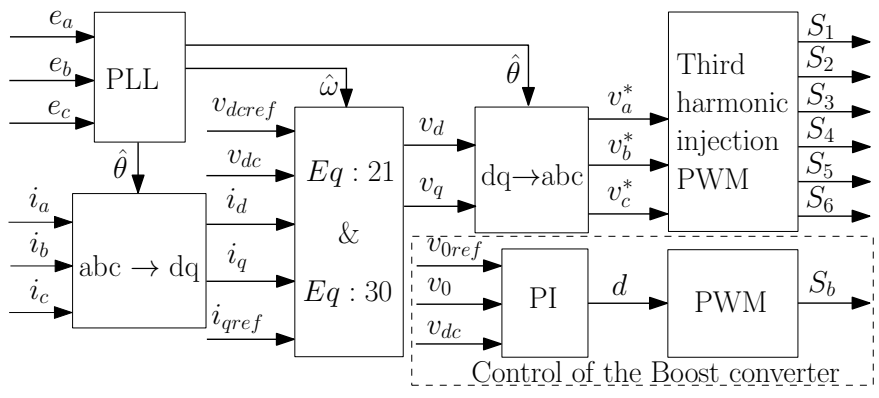

Fig. 4. Block diagram of the proposed NMPC scheme for a grid-connected $\mathrm{PV}$ inverter.

\section{CONTROLlER IMPLEMENTATION}

As mentioned earlier, the focus of the paper is to implement a continuous nonlinear MPC with a disturbance observer in real time to control the grid-tied inverter of the PV system. The control block diagram is briefly shown in Fig. 4. Unlike the cascaded structure, the proposed scheme provides directly the $d q$ components of the voltage, and the $d$-axis current is considered as an internal dynamics that has to be limited during the transients, so that the current does not exceed the peak value. This can be achieved by choosing adequately the DC-link voltage reference. As the reference $v_{d c r e f}$ can be twice differentiable, the DC-link voltage reference trajectory is realized by a second-order linear filter with slow dynamics, so that the current does not go above its maximal value during transients. The limitation of the current, during transients, can also be achieved by using a ramp reference signal with an appropriate choice of the slope. However, such approaches may limit the transient performances of the controller and may not guarantee current limitation under abrupt disturbance if the reference signal is not appropriately designed. The three-phase voltage commands $v_{a}^{*}, v_{b}^{*}$ and $v_{c}^{*}$ are realized by using the third harmonic injection PWM approach related to min-max technique. To consider the real dynamics of the PV system, a boost converter is also controlled in real time to track the desired power. Once the input $v_{d c}$ of the boost converter is regulated to its reference $v_{d c r e f}$, the $\mathrm{PV}$ voltage $v_{0}$ can be adjusted by controlling the switch duty-ratio $d$ of the boost converter. This is done by a simple PI controller, which is not detailed here, as it is beyond the scope of this paper. The voltage reference $v_{0 r e f}$ for the PI controller is chosen to correspond to the desired operating point of the PV array.

\section{EXPERIMENTAL RESULTS}

Many scenarios were performed to experimentally verify the system performances and the effectiveness of the proposed controller such as tracking performance, robustness, and disturbance rejection. To practically implement the proposed approach, the control gains are designed, so that the electric current loop has faster response than the DC-link voltage loop. Hence, following (20), the predictive times $T_{1}$ and $T_{2}$ are set to 1 and $10 \mathrm{~ms}$, respectively, which are much larger than the control period of $0.08 \mathrm{~ms}$. The disturbance observer is designed separately, and the observer gains $\mu_{d}, \mu_{q}$ and $\mu_{v}$ are chosen equal to -0.1 . Here, the disturbance observer is designed to ensure a trade-off between smooth estimate and fast disturbance estimation.

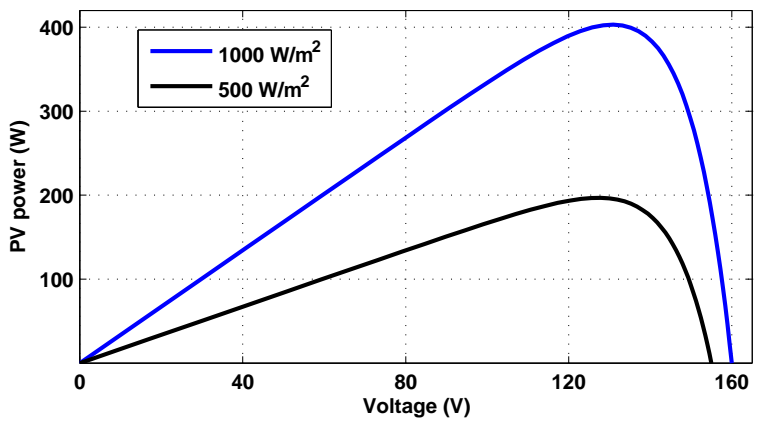

Fig. 5. V-P characteristics under two different solar irradiations.

The profile of the power injected into the grid is set to mimic the real dynamics of PV system by considering the changes of atmospheric conditions such as temperature and solar irradiation $\mathrm{Wm}^{-2}$. In this work, the temperature is chosen equal to its standard value and only solar irradiation 
change is considered because it can significantly affect the PV power as shown in Fig. 5. The results are demonstrated using 3 scenarios under model uncertainty. In the experimental work, the Tektronix high voltage differential probe (P5 200) and the Tektronix current probe amplifier (TCP A300) were used in conjunction with the Tektronix 4104B-L digital phosphor oscilloscope.

\section{A. Scenario-1:Start-up Phase-Tracking Performance With Only Grid-Tied Inverter Under Model Uncertainty}

First test deals with trajectory tracking for the DC-link voltage. In practical application, this test can represent the start-up phase. In this case, the PV system is disconnected from the DC-link capacitor via a Triple-Pole-Single-Throw (TPST) knife switch as shown in Fig. 1. The inductance $L$, the capacitance $C$ and the $d$-axis component $e_{d}$ of the grid voltage were incorrectly set in the controller at $t=0$, and their values are chosen equal to $50 \%$ of the actual one. As a result, the disturbances are caused by both the model uncertainty and the unavoidable PWM offset. This is because the inverter cannot provide exactly the three-phase voltages commands. At the start-up, the inverter works as a rectifier, and at $t=1 \mathrm{~s}$, a step change is made to regulate the DC-link voltage to a reference of $165 \mathrm{~V}$, which is greater than the open-circuit voltage, as it can be observed from Fig. 5 .

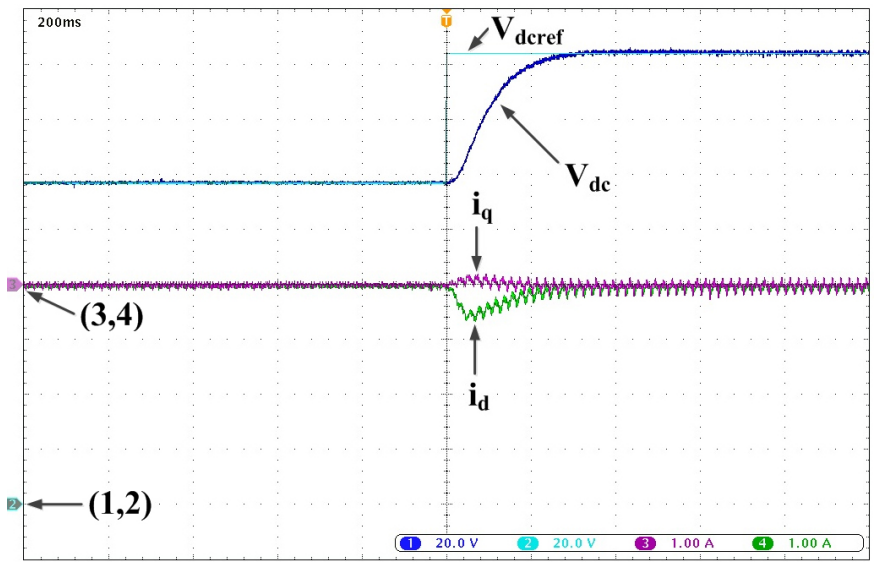

Fig. 6. DC-link voltage trajectory tracking and $d q$-axis currents (Scenario-1)

As shown in Fig. 6, the controller has proved to be effective regarding trajectory tracking. It is worth nothing that the actual signal reference is provided by a second-order linear filter with a slow dynamics in order to limit the current during transients (see the Appendix C). Thus, the actual reference signal is not plotted here, and only the input of the filter is shown. This is because the DC-link voltage almost coincides with the reference signal. From the results, it is clear that the $q$-axis component of the current is maintained equal to zero, and the $d$-axis current is well controlled during transients. The current ripples are due to the switching action of the PWM.

\section{B. Scenario-2: Normal Operation Under Model Uncertainty}

In order to investigate the disturbance rejection capability, the complete system with a $400 \mathrm{~W}$ PV array using a PV emulator, a conventional boost converter, and a grid-tied inverter is considered in this experiment. In addition, the parameters of the system are incorrectly set in the controller in the same manner as for the previous test. The V-P characteristic of the PV emulator shown in Fig. 5 is designed such that the maximal power is approximately $400 \mathrm{~W}$ under standard conditions with the designed capacitor's voltage and inductor's current limit. Fig. 5 depicts the V-P characteristic of the PV emulator under irradiation drop from 1000 to $500 \mathrm{Wm}^{-2}$, and Fig. 7 shows the profile of the solar irradiation used for investigating the disturbance rejection capability. The experimental results are shown in Figs. 8, 9 and 10, from where it can be observed that the steady-state error is quickly removed, and the proposed approach is able to maintain the system at the desired operating point even with the presence of external disturbance and model uncertainty. It can also be seen that the $q$-axis current is well controlled and it is insensitive to the disturbances. From Fig. 9, it can be found that the estimate quickly converges to a stable steady-state and modifies the control to compensate for the disturbances. It can also be observed that the estimate of the unmatched disturbance $b_{v}$ is smooth although the PV current contains high frequency component. This confirmed the fact that the disturbance observer can act as a low-pass filter. From Fig. 10, it can be concluded that the grid-connected photovoltaic system operates at unity power factor as expected.

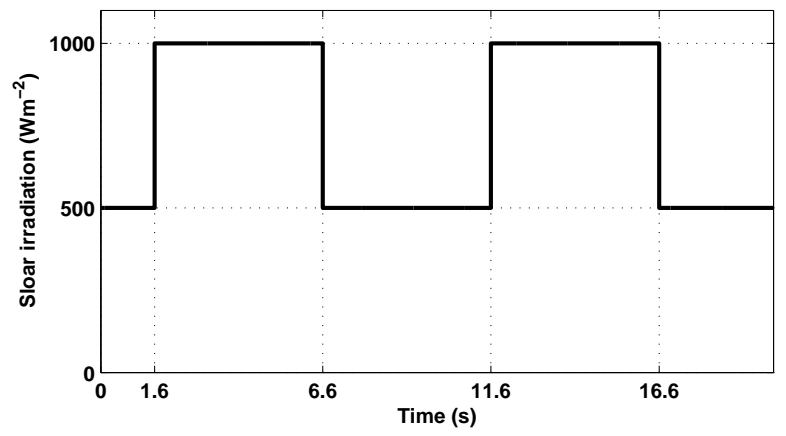

Fig. 7. Profile of the solar irradiation (Scenario-2).

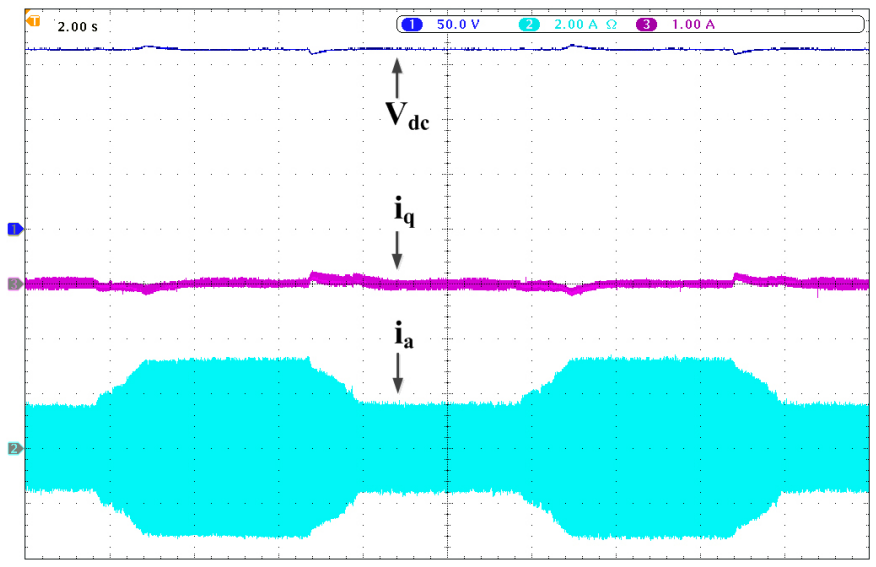

Fig. 8. DC-link voltage, $q$-axis current and Line filter current $i_{a}$ under gridinterlinked PV inverter (Scenario-2). 


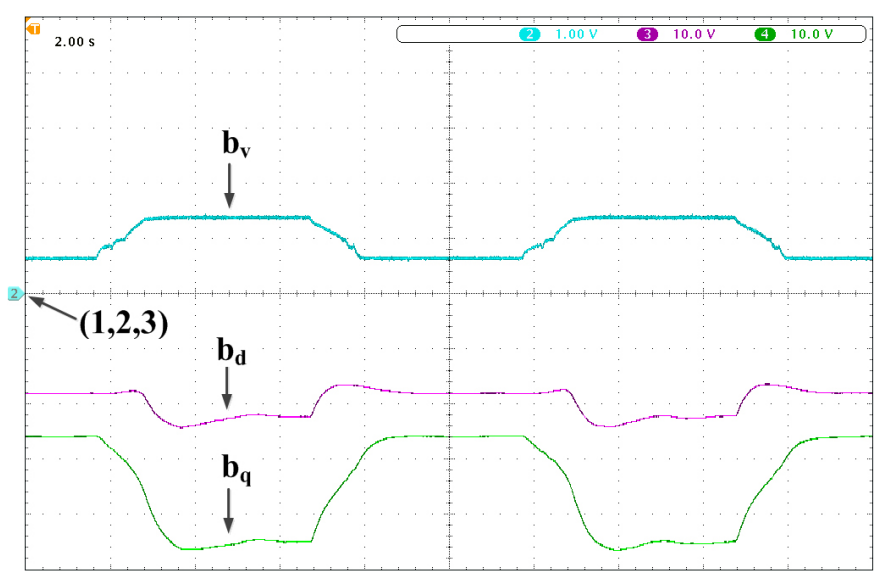

Fig. 9. Disturbances estimation $b_{d}, b_{q}$ and $b_{v}$ under grid-interlinked PV inverter (Scenario-2).

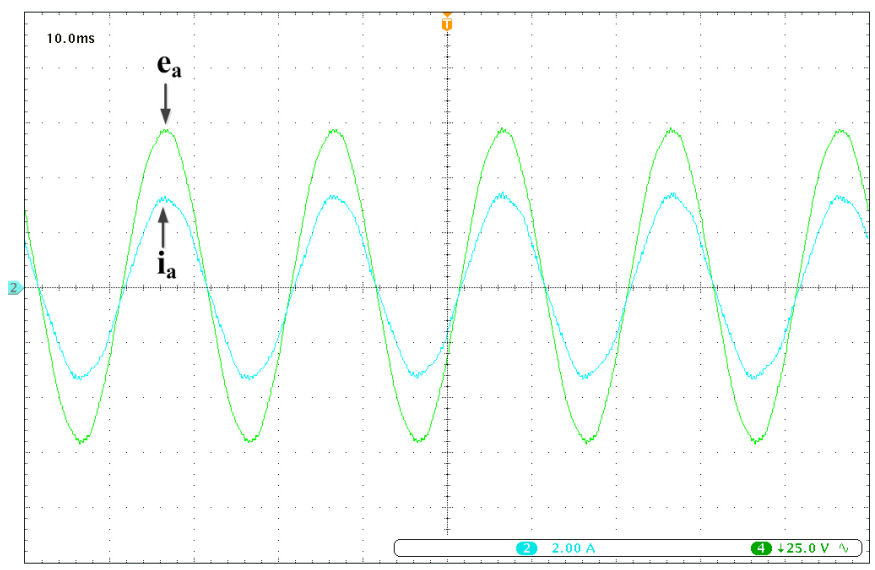

Fig. 10. Line filter current $i_{a}$ and grid voltage $e_{a}$ under grid-interlinked PV inverter (Scenario-2).

\section{Scenario-3: Performance Evaluation Under Abrupt and Varying Disturbance, and Model Uncertainty}

To further investigate the disturbance rejection capability, an abrupt disturbance was applied to the system by suddenly disconnecting the PV system from the grid. The disconnection test can be used to impersonate emergency scenario. This can be done by a sudden OFF switching of the TPST switch shown in Fig. 1. In addition, the inductance $L$ and the capacitor $C$ were incorrectly set in the controller, and their values are chosen equal to $150 \%$ of the actual one. The solar irradiation was fixed to $1000 \mathrm{Wm}^{-2}$. The instant of disconnection of the PV system from the grid can be observed in Fig. 11. From the results, it can be seen that the proposed approach is capable of rejecting abrupt disturbances, within a short time, which is mainly governed by the observer gain. The $q$-axis current is well controlled and is maintained equal to its reference despite the disturbance.

Another test was performed to show the effect of the disturbance on the THD of the line current, and the table I shows the corresponding results. In this test, the Fluke i310s AC/DC current clamps were used in conjunction with the Fluke 435-II power quality analyzer to measure the total harmonic current distortion. From the results, it can be observed that the THD is below $5 \%$ over large range of operating points, which meets the host grid requirements. More specifically, the THD decreases with decreasing the power because the current harmonics flowing through the line filter are much more dominant at low power.

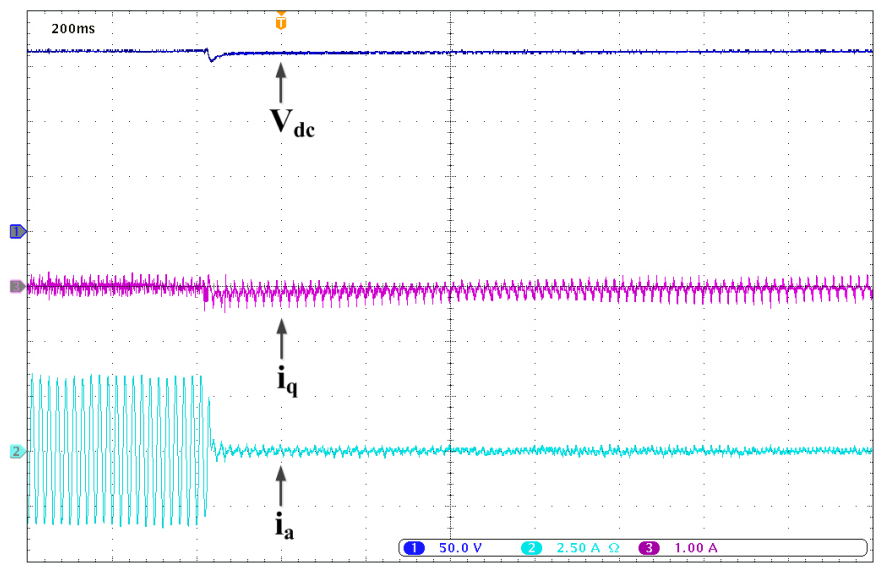

Fig. 11. DC-link voltage, $q$-axis current and Line filter current $i_{a}$ under grid-interlinked PV inverter (Scenario-3).

TABLE I

THE EFFECT OF THE PV OUTPUT POWER (DISTURBANCE) ON THE LINE CURRENT THD

\begin{tabular}{|l|l|l|l|l|}
\hline PV output power (W) & 390 & 290 & 220 & 135 \\
\hline Current THD (\%) & 2.1 & 2.3 & 2.8 & 4.4 \\
\hline
\end{tabular}

\section{CONCLUSION}

This paper presents a real-time implementation of a robust CNMPC for a grid-interlinked photovoltaic inverter. The case where the system is subjected to either model/plant mismatch or external perturbation is investigated to enhance the robustness of the closed-loop system. To this end, the existing nonlinear disturbance observer-based control is combined with CNMPC to compensate for the disturbances. Furthermore, global stability analysis of the composite controller is provided. It is shown that zero steady-state error is guaranteed in the presence of unknown matched and unmatched disturbances. The proposed approach is experimentally applied to the system under investigation to illustrate its effectiveness regarding trajectory tracking, stability, and disturbance rejection using three different realistic scenarios.

To the best of our knowledge, real-time implementation of the proposed approach has not been considered elsewhere. Thereby, the method proposed in this paper provides practitioners with an alternate way in improving disturbance rejection for a grid-interlinked PV inverter under a wide operating range.

\section{APPENDIX A \\ DERIVATION OF THE CNMPC IN SECTION III}

The predicted error $e(t+\tau)$ can be approximated by using Taylor series expansion up to the relative degree. Considering the relative degree of each output, one can write

$$
e(t+\tau)=\left[\begin{array}{ll}
e_{1}(t+\tau) & e_{2}(t+\tau)
\end{array}\right]^{T}=\Psi(\tau) \mathrm{E}(t)
$$


where

$$
\Psi(\tau)=\left[\begin{array}{c}
\Psi_{1}(\tau) \\
\Psi_{2}(\tau)
\end{array}\right]=\left[\begin{array}{ccccc}
1 & \tau & 0 & 0 & 0 \\
0 & 0 & 1 & \tau & \tau^{2} / 2 !
\end{array}\right]
$$

and

$$
\mathrm{E}(t)=\left[\begin{array}{lllll}
e_{1}(t) & \dot{e}_{1}(t) & e_{2}(t) & \dot{e}_{2}(t) & \ddot{e}_{2}(t)
\end{array}\right]^{T}
$$

Substituting (38) into (8), we obtain the approximate cost function

$$
\Im=\frac{1}{2} E(t)^{T} \Upsilon\left(T_{1}, T_{2}\right) E(t)
$$

where

$$
\Upsilon\left(T_{1}, T_{2}\right)=\int_{0}^{T_{1}} \Psi_{1}(\tau)^{T} \Psi_{1}(\tau) d \tau+\int_{0}^{T_{2}} \Psi_{2}(\tau)^{T} \Psi_{2}(\tau) d \tau
$$

Combining (12)-(14) yields

$$
\left\{\begin{array}{l}
\dot{e}_{1}(t)=\dot{y}_{r 1}(t)-L_{f} h_{1}(x)-L_{g_{1}} h_{1}(x) u-L_{g_{2}} h_{1}(x) b \\
\dot{e}_{2}(t)=\dot{y}_{r 2}(t)-L_{f} h_{2}(x)-L_{g_{2}} h_{2}(x) b
\end{array}\right.
$$

and

$$
\begin{gathered}
\ddot{e}_{2}(t)=\ddot{y}_{r 2}(t)-L_{f}^{2} h_{2}(x)-L_{g_{1}} L_{f} h_{2}(x) u-\dot{b}_{v} / C \\
-L_{g_{2}} L_{f} h_{2}(x) b
\end{gathered}
$$

Now, substitute (43)-(44) into (41), and invoke the necessary condition of optimality to get the closed-form of the CNMPC defined by (15).

\section{APPENDIX B}

\section{PARAMETERS OF THE GRID-INTERLINKED PV INVERTER}

For a given DC-link voltage $v_{d c}$, the maximum voltage that the two level inverter can produce, using a third harmonic injection approach, is given by

$$
\sqrt{v_{d}^{2}+v_{q}^{2}}=\frac{v_{d c}}{\sqrt{3}}
$$

From (1), it follows that, at steady state, the amplitude voltage $E_{\max }$ satisfies

$$
\sqrt{v_{d}^{2}+v_{q}^{2}}=\sqrt{\left(R i_{d}-L \omega i_{q}+E_{\max }\right)^{2}+\left(R i_{q}+L \omega i_{d}\right)^{2}} \leq \frac{v_{d c}}{\sqrt{3}}
$$

The $d$-axis current can be expressed as a function of the active power $P$, injected into the grid, as follows

$$
i_{d}=\frac{2 P}{3 E_{\max }}
$$

Thus, if there is no power exchange between the PV system and the grid, i.e., $i_{d}=0$ and $i_{q}=0$, then the following inequality must hold for the grid amplitude voltage $E_{\max }$

$$
E_{\max } \leq \frac{v_{d c}}{\sqrt{3}}
$$

Therefore, for the system under study, the choice of the grid voltage amplitude mainly depends on the DC-link voltage value. Such a parameter is limited by many factors. Among them, the DC-link capacitor's voltage limit, the PV panel output voltage, the inductor current ripples of the dc-dc converter which increase with increasing the DC-link voltage for a given inductance and a limited switching frequency.

On the other hand, by neglecting the resistance $R$ and combining (46) with (47), it can be shown that, under unity power factor operation, i.e., $i_{q}=0$, the following inequality must hold

$$
P \leq \frac{3 E_{\max }}{\sqrt{2} L \omega} \sqrt{\left(\frac{v_{d c}^{2}}{6}-\frac{E_{\max }^{2}}{2}\right)}
$$

Inequality (49) implies that the active power $P$ is limited by the value of the voltage amplitude $E_{\max }$. Nevertheless, as the right-hand side of the inequality (49) is a concave function with respect to $E_{\max }$, one can choose $E_{\max }$ to correspond to the maximum value of $P$. Such a value is determined as

$$
\frac{\partial\left(\frac{3 E_{\max }}{\sqrt{2} L \omega} \sqrt{\left(\frac{v_{d c}^{2}}{6}-\frac{E_{\max }^{2}}{2}\right)}\right)}{\partial E_{\max }}=0 \rightarrow E_{\max }=\frac{v_{d c}}{\sqrt{6}}
$$

Substituting (50) in (49) yields

$$
P \leq P_{\max }=\frac{v_{d c}^{2}}{4 L \omega}
$$

For a given line inductance $L$, the above inequality is used as a basis to define the V-P characteristic of the PV emulator for the purpose of testing the proposed controller.

The parameter values of the grid-interlinked PV inverter system are summarized in Table II.

TABLE II

PARAMETERS OF THE GRID-INTERLINKED PV INVERTER

\begin{tabular}{|l|l|}
\hline Maximum power of PV unit (W) & 400 \\
\hline DC link voltage (V) & 165 \\
\hline Line-to-line grid voltage (V) & 84.8 \\
\hline Inverter inductance (mH) & 60 \\
\hline DC link capacitor $(\mathrm{mF})$ & 1.052 \\
\hline Boost inductance $(\mathrm{mH})$ & 0.8 \\
\hline Frequency $(\mathrm{Hz})$ & 50 \\
\hline
\end{tabular}

\section{APPENDIX C \\ SECOND-ORdER Linear FiLTER}

The DC-link voltage is realized using the following secondorder linear filter

$$
H(s)=\frac{\omega_{0}^{2}}{s^{2}+2 \xi \omega_{0} s+\omega_{0}^{2}}
$$

where $\xi=1$ and $\omega_{0}=20 \mathrm{rad} / \mathrm{s}$

\section{REFERENCES}

[1] C. E. Garcia, D. M. Prett, and M. Morari, "Model predictive control: theory and practice-a survey," Automatica, vol. 25, no. 3, pp. 335-348, 1989.

[2] S. Kouro, P. Cortés, R. Vargas, U. Ammann, and J. Rodríguez, "Model predictive control-a simple and powerful method to control power converters," IEEE Trans. Ind. Electron., vol. 56, no. 6, pp. 1826-1838, 2009.

[3] J. D. Barros, J. F. A. Silva, and É. G. Jesus, "Fast-predictive optimal control of npc multilevel converters," IEEE Trans. Ind. Electron., vol. 60, no. 2, pp. 619-627, 2013.

[4] J. Rodriguez, M. P. Kazmierkowski, J. R. Espinoza, P. Zanchetta, H. Abu-Rub, H. A. Young, and C. A. Rojas, "State of the art of finite control set model predictive control in power electronics," IEEE Trans. Ind. Informat., vol. 9, no. 2, pp. 1003-1016, 2013. 
[5] D.-K. Choi and K.-B. Lee, "Dynamic performance improvement of ac/dc converter using model predictive direct power control with finite control set," IEEE Trans. Ind. Electron., vol. 62, no. 2, pp. 757-767, Feb 2015.

[6] V. Yaramasu, M. Rivera, B. Wu, and J. Rodriguez, "Model predictive current control of two-level four-leg inverters-part I: concept, algorithm, and simulation analysis," IEEE Trans. Power Electron., vol. 28, no. 7, pp. 3459-3468, 2013.

[7] M. Rivera, V. Yaramasu, J. Rodriguez, and B. Wu, "Model predictive current control of two-level four-leg inverters-part II: experimental implementation and validation," IEEE Trans. Power Electron., vol. 28, no. 7, pp. 3469-3478, 2013.

[8] J. Scoltock, T. Geyer, and U. Madawala, "Model predictive direct power control for grid-connected npc converters," IEEE Trans. Ind. Electron., vol. 62, no. 9, pp. 5319-5328, 2015.

[9] J. Hu, J. Zhu, and D. G. Dorrell, "Model Predictive Control of GridConnected Inverters for PV Systems With Flexible Power Regulation and Switching Frequency Reduction," IEEE Trans. Ind. Appl., vol. 51, no. 1, pp. 587-594, 2015.

[10] Z. Song, C. Xia, and T. Liu, "Predictive current control of three-phase grid-connected converters with constant switching frequency for wind energy systems," IEEE Trans. Ind. Electron., vol. 60, no. 6, pp. 24512464, 2013.

[11] M. Shadmand, R. Balog, and H. Abu-Rub, "Model predictive control of pv sources in a smart de distribution system: Maximum power point tracking and droop control," IEEE Trans. Energy Conv., vol. 29, no. 4, pp. 913-921, Dec 2014.

[12] V. Yaramasu, B. Wu, S. Alepuz, and S. Kouro, "Predictive control for low-voltage ride-through enhancement of three-level-boost and npcconverter-based pmsg wind turbine," IEEE Trans. Ind. Electron., vol. 61 no. 12, pp. 6832-6843, Dec 2014.

[13] J. Espi Huerta, J. Castello-Moreno, J. Fischer, and R. Garcia-Gil, "A synchronous reference frame robust predictive current control for threephase grid-connected inverters," IEEE Trans. Ind. Electron., vol. 57, no. 3, pp. 954-962, March 2010.

[14] V. Yaramasu and B. Wu, "Model predictive decoupled active and reactive power control for high-power grid-connected four-level diode-clamped inverters," IEEE Trans. Ind. Electron., vol. 61, no. 7, pp. 3407-3416, July 2014.

[15] P. E. Kakosimos, A. G. Kladas, and S. N. Manias, "Fast photovoltaicsystem voltage-or current-oriented mppt employing a predictive digital current-controlled converter," IEEE Trans. Ind. Electron., vol. 60, no. 12 pp. 5673-5685, 2013.

[16] M. Soroush and C. Kravaris, "A continuous-time formulation of nonlinear model predictive control," Int. J. Control, vol. 63, no. 1, pp. 121-146, 1996.

[17] P. Lu, "Nonlinear predictive controllers for continuous systems," J. Guid. Control Dynam., vol. 17, no. 3, pp. 553-560, 1994.

[18] P. Lu, "Optimal predictive control of continuous nonlinear systems," Int. J. Control, vol. 62, no. 3, pp. 633-649, 1995.

[19] P. Lu, "Approximate nonlinear receding-horizon control laws in closed form," Int. J. Control, vol. 71, no. 1, pp. 19-34, 1998.

[20] W.-H. Chen, D. J. Ballance, P. J. Gawthrop, J. J. Gribble, and J. O'Reilly, "Nonlinear pid predictive controller," IEE Proc. Control Theory Appl., vol. 146, no. 6, pp. 603-611, 1999.

[21] W. Feng, J. O'Reilly, and D. Ballance, "Mimo nonlinear pid predictive controller," IEE Proc. Control Theory Appl., vol. 149, no. 3, pp. 203 208, 2002.

[22] W.-H. Chen, D. J. Ballance, and P. J. Gawthrop, "Optimal control of nonlinear systems: a predictive control approach," Automatica, vol. 39 , no. 4, pp. 633-641, 2003.

[23] J. Yang, W. Zheng, S. Li, B. Wu, and M. Cheng, "Design of a prediction accuracy enhanced continuous-time mpc for disturbed systems via a disturbance observer," IEEE Trans. Ind. Electron., vol. 62, no. 9, pp. 5807-5816, 2015.

[24] R. Errouissi, M. Ouhrouche, W.-H. Chen, and A. M. Trzynadlowski, "Robust cascaded nonlinear predictive control of a permanent magnet synchronous motor with antiwindup compensator," IEEE Trans. Ind. Electron., vol. 59, no. 8, pp. 3078-3088, 2012.

[25] P. J. Gawthrop, H. Demircioglu, and I. I. Siller-Alcala, "Multivariable continuous-time generalised predictive control: A state-space approach to linear and nonlinear systems," IEE Proc. Control Theory Appl, vol. 145 , no. 3, pp. 241-250, 1998.

[26] F. Delfino, G. Denegri, M. Invernizzi, and R. Procopio, "Feedback linearisation oriented approach to $\mathrm{q}-\mathrm{v}$ control of grid connected photovoltaic units," IET Renew. Power Gen., vol. 6, no. 5, pp. 324-339, Sept 2012
[27] M. Mahmud, H. Pota, M. Hossain, and N. Roy, "Robust partial feedback linearizing stabilization scheme for three-phase grid-connected photovoltaic systems," IEEE J. Photovolt., vol. 4, no. 1, pp. 423-431, Jan 2014.

[28] D. Lalili, A. Mellit, N. Lourci, B. Medjahed, and E. Berkouk, "Input output feedback linearization control and variable step size mppt algorithm of a grid-connected photovoltaic inverter," Renew. Energy, vol. 36, no. 12 , pp. 3282-3291, 2011

[29] C. Wang, X. Li, L. Guo, and Y. W. Li, "A nonlinear-disturbanceobserver-based dc-bus voltage control for a hybrid ac/dc microgrid," IEEE Trans. Power Electron., vol. 29, no. 11, pp. 6162-6177, Nov 2014.

[30] Y. I. Son, I. H. Kim, D. S. Choi, and H. Shim, "Robust cascade control of electric motor drives using dual reduced-order pi observer," IEEE Trans. Ind. Electron., vol. 62, no. 6, pp. 3672-3682, 2015.

[31] R. Errouissi, M. Ouhrouche, W.-H. Chen, and A. M. Trzynadlowski, "Robust nonlinear predictive controller for permanent-magnet synchronous motors with an optimized cost function," IEEE Trans. Ind. Electron., vol. 59, no. 7, pp. 2849-2858, 2012.

[32] V. Kaura and V. Blasko, "Operation of a phase locked loop system under distorted utility conditions," IEEE Trans. Ind. Appl., vol. 33, no. 1, pp. 58-63, Jan 1997.

[33] S.-K. Chung, "A phase tracking system for three phase utility interface inverters," IEEE Trans. Power Electron., vol. 15, no. 3, pp. 431-438, 2000.

[34] H. K. Khalil, Nonlinear systems. Prentice hall Upper Saddle River, 2002, vol. 3.

[35] J. Yang, S. Li, and X. Yu, "Sliding-mode control for systems with mismatched uncertainties via a disturbance observer," IEEE Trans. Ind. Electron., vol. 60, no. 1, pp. 160-169, 2013.

[36] A. Isidori, Nonlinear Control Systems: An Introduction(3rd edn). Springer-Verlag, 1995.

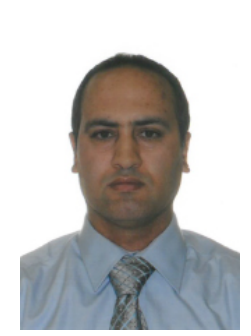

Rachid Errouissi (M'15) received the B.Sc. degree in electronics from the Faculty of Sciences and Technology of Mohammedia, Morocco, in 1998; the M.Sc. degree in power electronics from Ecole Mohammadia dŠingnieurs in Rabat, Morocco, in 2001; the double M.Sc. degrees in electrical engineering and in automation and system engineering from University Claude Bernard, Lyon, France, in 2002 and 2004; and the Ph.D. degree in electrical engineering from the University of Quebec, Chicoutimi, QC Canada, in 2010. From 2011 to 2014, he worked as a Postdoctoral Researcher with the Department of Electrical and Computer Engineering, University of New Brunswick. Since 2014, he has been with the Petroleum Institute, Abu Dhabi, UAE, where he is currently conducting research works in renewable energy and advanced control systems. His area of interests include advanced control, nonlinear control, electric machines and drives, and renewable energy conversion systems. He is a registered Professional Engineer in the province of New Brunswick, Canada.

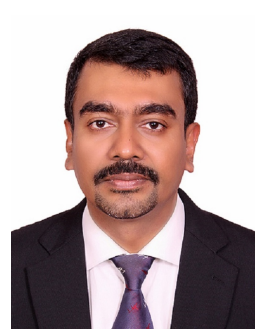

S. M. Muyeen (S'03-M'08-SM'12) received his B.Sc. Eng. Degree from Rajshahi University of Engineering and Technology (RUET), Bangladesh formerly known as Rajshahi Institute of Technology, in 2000 and M. Sc. Eng. and Dr. Eng. Degrees from Kitami Institute of Technology, Japan, in 2005 and 2008, respectively, all in Electrical and Electronic Engineering. His $\mathrm{PhD}$ research work focused on wind farm stabilization from the viewpoint of LVRT and frequency fluctuation. After completing his Ph.D. program he worked as a Postdoctoral Research Fellow under the versatile banner of Japan Society for the Promotion of Science (JSPS) from 2008-2010 at the Kitami Institute of Technology, Japan. At the present, he is working as Associate Professor in Electrical Engineering Department at the Petroleum Institute, Abu Dhabi. His research interests are power system stability and control, electrical machine, FACTS, energy storage system (ESS), Renewable Energy, and HVDC system. He has been a Keynote Speaker and an Invited Speaker at many international conferences, workshops, and universities. He has published over 150 articles in different journals and international conferences. He has published five books as an author or editor. Dr. Muyeen is the senior member of IEEE. 


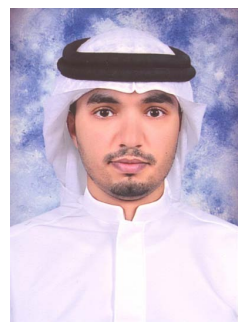

Ahmed Al-Durra (S'07-M'10-SM'14) received the B.S., M.S., and $\mathrm{PhD}$ in Electrical and Computer Engineering from the Ohio State University in 2005, 2007, and 2010, respectively. For his M. Sc. degree, he investigated the application of several nonlinear control techniques on automotive traction PEM fuel cell systems. He conducted his $\mathrm{PhD}$ research at the Center for Automotive Research in the Ohio State University on the applications of modern estimation and control theories to automotive propulsion systems. At the present, he is working as Associate Professor in Electrical Engineering Department and the Downstream Research Coordinator of the PI Research Center at the Petroleum Institute, Abu Dhabi. His research interests are application of estimation and control theory in power system stability, Micro and Smart Grids, renewable energy, and process control. He has published over 60 scientific articles in Journals and International Conferences. Dr. Ahmed has successfully accomplished several research projects at international and national levels. He is the co-founder of Renewable Energy Laboratory at the Petroleum Institute, Senior Member in IEEE, and a Member in ASME.

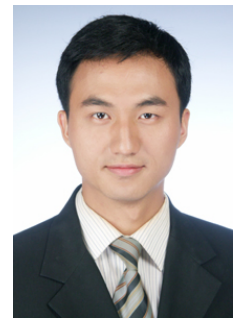

Siyu Leng (S'09-M'14) received his B.Sc. from Tongji University in 2006. He was granted a full scholarship from the Florida State University to study in the U.S.A. During his Ph.D. study, he joined the Center for Advanced Power Systems (CAPS) as a graduate research assistant under the supervision of Prof. David A. Cartes. His research at CAPS was focused on power quality, especially active power filters. After receiving his Ph.D. in 2012, he went back to China to work as a research engineer at State Grid Electric Power Research Institute. From May 2013, he has been working as a postdoctoral fellow at University of Alberta under the supervision of Prof. John Salmon. His research at University of Alberta was focused on power electronics, especially induction motor drives. He joined Petroleum Institute as a research/teaching associate in October, 2014. His research interests includes: renewable energy, power electronics, motor drive, power quality, power system analysis. 